\title{
A gradual advancement of the extraction and cleanup techniques for residue analysis of pesticides in food matrices and liquid products: a review
}

\author{
Sandeep K. Singh ${ }^{1}$, Rajneesh Dwevedi ${ }^{2}$, Pooja Deopa ${ }^{3}$, Vinoy Krishna ${ }^{4}$ \\ ${ }^{1}$ Zoology Department, Ramjas College, University of Delhi. \\ ${ }^{2,4}$ Department of Life Sciences, Lady Irwin College, University of Delhi.
}

\begin{abstract}
In this review we have discussed the methods which have been used for extraction and cleanup during multi residue methods (MRM) of pesticides in food matrices and liquid food products. Single residue method (SRM) is used for the analysis of single pesticide residue in matrix. Now days it is very common to find pesticides in the food products because of the increased use of pesticides for the protection and better productivity of food materials. Different types of pesticides are used on the plants and food materials. There is a gradual advancement in the multi pesticide residue analysis of food and liquid food products so that the use of pesticides can be monitored. In this concern there are methods which are used for the single pesticide residue analysis and multi pesticide residue analysis. The development of residue analysis methods depends on the properties of matrices and pesticides. In recent time the advancement in techniques and instrumentation has contributed to great extent in the improvement of residue analysis. Soxlet extraction (SOX) is still in use for MRM. Liquid- liquid extraction (LLE) is applied in the pesticide residue analysis of commodities with highmoisture.. Earlier the process of residue analysis were long and tiring and involved the use of larger quantity of hazardous solvent and other chemicals but the advanced methods are more convenient and lesser in cost.
\end{abstract}

Key words- MRM, SRM, SOX, LLE, SPE, SPME, MSPD, SFE, ASE.

\section{Introduction}

The use of pesticides for the security of crops and food products is very common these days. This information also carries the fact that higher or lower quantity pesticides are found in food products. The availability of pesticides in food products can be controlled by monitoring the use of pesticides.

Residue analysis of food matrices involves several steps. There are many extraction and cleanup procedures used by different workers to determine the amount of pesticides in different matrices.

There are two general analytical approaches to determine residues in food and environmental samples-

1-Single Residue Methods (SRM) - In this method a single pesticide and its metabolites are quantitatively determined in the sample.

2-Multiresidue Methods (MRM) - This approach is applied to detect and quantify more than one pesticide in more than one food or environmental samples. MRMs are generally used by government agencies for monitoring that which pesticide is present in the given sample and how much the actual amount of that pesticide. Screening and quantitation are two purposes of MRMs. In multi residue methods mainly gas or liquid chromatography are used ${ }^{1}$.

In this analysis of sample the analytes are extracted from matrix and then through cleanup process coextractives are removed. Many industrial organizations, regulatory bodies and laboratories are involved in this type of work. Basically the work of all these organizations benefits in monitoring the usage of pesticides. The selection of multi reside method depends on many aspects like trueness, precision, sensitivity, ease of use, low cost and labour, occupational and environmental friendliness, small space requirements, and minimum glassware and material needs ${ }^{2}$.

\section{1-TECHNIQUES OF EXTRACTION AND CLEANUP}

Polarity of analyte and the solubility of different pesticides in water is an important consideration in analytical procedures. The use of solvents and chemicals depends also on the property of food matrix. Matrices with fats above $2 \%$ are considered fatty. Water content is important for non fatty food products ${ }^{3,4}$.

Fruit, vegetable and cereal samples are usually blended with organic solvent alone, solvent mixtures, water or $\mathrm{pH}$-adjusted water (blending extraction). Acetone, acetonitrile $(\mathrm{MeCN})$, methanol and ethyl acetate are the most used organic solvents in the extraction of herbicides from food ${ }^{5}$.

\section{1- Purge and trap extraction}


This system was developed for analysis of volatile organic compounds (VOCs) in polluted water. The VOCs of interest are those that appear on the EPA priority list ${ }^{6}$ as purgable organics: 31 solvents (toluene, benzene, carbon tetrachloride, trichloroethylene etc.), monomers (bischloromethyl ether, vinyl chloride, acrylonitrile etc.), chlorofluorocarbons (trichlorofluoromethane, dichlorofluoromethane) and miscellaneous compounds (chlorobenzene, ethylbenzene, acrolein etc.), including at least one pesticide (methyl bromide). Purge and trap analysis usually entails use of a commercial instrument that is attached to a flame ionization gas chromatograph, automated in its handling of a carousel of water samples. Following steps are included in this analysis-

(i) Purge- Nitrogen is bubbled through the aqueous sample to volatilize the compounds.

(ii) Trap- Purged VOCs are trapped from the exit nitrogen purging gas by sorption on a column of Tenax.

(iii) Desorb- Tenax sorbed VOCs are thermally desorbed with carrier gas from the flash heated Tenax, and flushed directly into a GC, or indirectly after first condensing the liberated VOCs in cryogenic trap or on the cooled front end of the GC column.

(iv) Gas chromatography- Discrete VOCs are resolved on a GC column.

Actually all of the Purge and Trap steps, and criteria by which data quality are assessed, are mandated in EPA methods for VOCs. ${ }^{7}$ These steps may also be used for analyzing pesticides and pesticide metabolites that are volatile enough to be purged from water; methyl bromide, methyl isothiocynate, ethylene oxide, and propylene oxide. ${ }^{8}$

\section{2- Soxhlet extraction (SOX)}

Soxhlet extraction with an organic solvent (e.g. hexane, acetone, dichioromethane, or methanol, methanol-water) is still used today as a MRM for pesticide analysis in solid matrices. The size of the system can vary, but the most common configurations use $100 \mathrm{ml}$ of solvent and 5-50 g of solid sample, with extractions lasting from 4 to $18 \mathrm{~h} .{ }^{9}$ In some cases, extraction by simply standing the whole grain (rice, maize, sunflower seed or pea) for $48 \mathrm{~h}$ generally resulted in similar extraction levels of Carbaryl as those obtained after grinding and SOX. However, the latter procedure removed the pesticide more rapidly. ${ }^{10}$

\section{3- Liquid-liquid extraction ( $L L E)$}

LLE applies to liquid matrices. The non-polar solvents are n-hexane, benzene and ethyl acetate. The water-miscible solvents such as dichloromethane, methanol and acetone are employed for extraction of residues from high-moisture commodities. Sometimes solvents are combined to achieve the desired viscosity and solvency strength for the particular extraction. LLE extractors have large size, utilize a considerable amount of solvent and must be controlled in terms of stirring. Initial MRMs employed MeCN as the extraction solvent; the disadvantages of $\mathrm{MeCN}$ are its high price and toxicity. Later on, acetone (b.pt. $56.5^{\circ} \mathrm{C}$ ) was used instead of $\mathrm{MeCN}$ (b.pt. $81.6^{\circ} \mathrm{C}$ ) because it has higher volatility (which makes it easier to remove) in the most generally used method for analysis of pesticides in fruits and vegetables (Luke method) ${ }^{11}$, a disadvantage of which is the use of the environmental carcinogen dichloromethane. Extraction with ethyl acetate, as employed in the Swedish extraction procedure ${ }^{12}$, is less labor intensive with high yield. Because ethyl acetate (EtAc) is not completely miscible with water, no extra partition steps after extraction are needed because the excess sodium acetate added can remove water.

\section{4- Solid-phase extraction (SPE)}

Besides the use of LLE for the isolation and preconcentration of a particular component from the sample, the use of extraction in the liquid-solid phase system is growing, mainly for matrices with a high content of water. In literature it is frequently denoted as solid-phase extraction (SPE). SPE is a simple preparation technique based on the separation of liquid chromatography, where the solubility and functional group interactions of sample, solvent and sorbent are optimized to affect the retention and elution.

Moderately polar to polar analytes are extracted from non-polar solutions onto polar sorbents. Sorbents for normal phase are modified with cyano-, diol- or amino groups. Non-polar to-moderately polar analytes are extracted from polar solutions onto non-polar sorbents. Sorbents for reversed-phase are modified with octadecyl-, octyl-, cyclohexyl- or phenyl groups. Solid-phase extraction (SPE) with a mixture of RP-18-PolarPlus and cyano material was used to replace liquid-liquid partition step done with dichloromethane SPE ${ }^{13-17}$ may be carried out with several modifications, e.g., on columns (cartridges) or using membrane extraction discs. Most MRMs include a clean-up step using adsorption columns, in particular Florisil, aluminum oxide (alumina) and silica gel. Most adsorbent columns provide good clean-up only when they are eluted with solvent mixtures of low polarity, eluting less polar residues and leaving more polar co-extractives in the column. The more the eluting solvent polarity is increased, the greater will be the portion of interfering substances eluted and the less effective the clean-up will be. 
Florisil has gained the greatest attention of all the sorbents used in residue analysis. As Florisil retains some lipids preferentially ( $25 \mathrm{~g}$ Florisil with $3 \%$ water retains $1 \mathrm{~g}$ of fat), it is particularly well suited for the clean-up of fatty foods. When a Florisil column is eluted with solvent mixtures of low polarity, non-polar residues are recovered almost quantitatively. The eluates are very clean for GC electrochemical detection (ECD), nitrogenphosphorus detection (NPD), flame photometric detection (FPD) as well as for TLC with selective detection using the Hill reaction inhibition technique ${ }^{18}$. Major disadvantage is, however, that activity may vary from one batch to another. For the analysis plant material, Florisil has been recommended often in the framework of multi-residue procedures for fruits and vegetables. Florisil columns have been used for the clean-up of plant extracts by many authors ${ }^{19-28}$. In many cases, Florisil can be replaced by alumina, particularly for the analysis of fatty foods. Basic alumina decomposes some organophosphates, and some more polar pesticides are not (or not quantitatively) eluted from neutral or acidic alumina columns. Alumina columns were used for clean-up by Ambrus et al, ${ }^{29}$ Pestemer and Mann $^{30}$ and by Tekel et al, ${ }^{31}$. In general, silica gel is less efficient than alumina and does not adequately separate pesticides from plant co-extractives. Its importance in the analysis of plant material resides in the fractionation of certain residues according to their polarity without appreciable losses. Many authors ${ }^{32,22-27,33}$ have used silica gel columns or mini-columns for the clean-up of sample extract. Lores et al, ${ }^{34}$ used an improved silica gel clean-up method for organophosphorus pesticides. The method used a $3.5 \mathrm{~g}$ silica column pre-loaded with $1 \%$ acetic acid. The recoveries ranged from 92 to $101 \%$ for the eleven organophosphorus compounds tested. Mixed adsorbents have been used to combine the different properties of hydrophilic adsorbents and lipophilic carbon. Some of them have gained some importance, such as the silicacarbon column or mixtures of carbon with $\mathrm{MgO}$ and Celite. Di Muccio et al, ${ }^{35}$ used single-step solid matrix clean-up of vegetable extracts from different crops (lettuce, onion, strawberry, apple, yellow pepper, peach, tomato, broccoli, cauliflower and radish) for the determination of organophosphorus pesticide residues. An aqueous acetone extract of the sample $(15 \mathrm{ml}$ aliquot of the extract equivalent to $\mathrm{Ca} .5 \mathrm{~g}$ of the crop) was transferred onto the top of an Extrelut- 20 column filled with a macroporous Kieselguhr-type material. The column was eluted with four portions of light petroleum (b.pt. $40-60^{\circ} \mathrm{C}$ ), followed by four portions of dichioromethane-light petroleum $(1: 3, \mathrm{v} / \mathrm{v})$ to elute dimethoate. The recoveries were between 75 and $110 \%(0.1$ to $1.4 \mathrm{mg} / \mathrm{kg}$ ). Graphitized carbon black (GCB) is such a sorbent, being non-specific and generally of hydrophobic nature. Contrary to sorbents based on $\mathrm{SiO}_{2}$, these may be used without the $\mathrm{pH}$ of the treated solutions being taken into account. The GCB columns are extensively used in the analysis of pesticide residues in water. Co-extractives were removed with a double- layered SPE column, and graphitized carbon black and primary secondary amine (GCB/PSA) solid phase extraction cleanup cartridge for the determination of 180 pesticide residues in fruits and vegetables ${ }^{36}$. Solid-phase extraction (SPE) on a highly cross-linked polystyrene divinylbenzene column (LiChrolut EN) was used for clean-up and pre-concentration of the pesticides from the water-diluted acetone extracts. For most fruit and vegetable samples this partial clean-up was sufficient, but some of them with more co-extracting substances need further clean-up (cereals, spinach, carrots, etc.). Diethylaminopropyl (DEA) modified silica was used for efficient removal of interferences caused by various organic acids, sugars, etc. Many of the published methods for pesticide determination in fresh fruits and vegetables use a combination of two or more commercially available SPE columns for clean-up in the normalphase (NP) mode. Weak anion-exchange sorbents such as primary secondary amine (PSA), aminopropyl $\left(\mathrm{NH}_{2}\right)$, or diethylaminopropyl (DEA) modified silica are often used for clean-up of food samples together with strong anion-exchange sorbents (SAX, QMA). An improved variation of the methodology of Luke and co-workers which has been referred to as Luke II method, uses a pre-partition clean-up with a C 18 SPE column, an acetone - methylene chloride partition clean-up step and post-partition clean-up using strong and weak anionexchange SPE catridges (QMA and aminopropyl). Other SPE clean-up approaches include the combination of GCB (graphitized carbon black) and PSA columns ${ }^{37-38}$, the combination of C18, GCB and aminopropyl and the combination of GCB, PSA and SAX columns ${ }^{39}$. Because of difficulties with elution of certain planar or aromatic pesticides from GCB, only PSA was used for very efficient clean-up of acetonitrile extracts. There are fewer applications using reversed-phase (RI-SPE) for pre-concentration clean-up of pesticide residues from fruit and vegetable samples. Using RP-SPE, nonpolar to moderately polar analytes are extracted from polar solutions (e.g. aqueous) onto nonpolar sorbents, which include silica modified with octadecyl, octyl, cyclohexyl or phenyl groups, modified or non modified poly(styrenedivinylbenzene) (PS-DVB) resins and GCB ${ }^{40,41}$. For solid food samples the first step is the extraction of pesticides using water-miscible solvents, but dilution with water is required to facilitate the retention of pesticides onto RP sorbent such as $\mathrm{C}_{18} 8^{42-45}, \mathrm{GCB}^{44,46-47}$ or polymeric sorbents $^{47-50}$. The dispersive-SPE with PSA was effectively used to remove many polar matrix components, such as organic acids, certain polar pigments, and sugars, to some extent from the food extracts ${ }^{51}$.

\section{5- Solid-phase micro-extraction (SPME)}

In the early 1990s, SPME was introduced as a rapid and simple procedure of extraction with a great capacity of concentration, without the need for any organic solvent, and the convenience of automation ${ }^{52}$. It 
allows the extraction and concentration to be focused in a single step. SPME consists of two separate steps: an extraction (retention of the analytes on the stationary phase) and a desorption step ${ }^{53}$. Both steps must be optimized for a successful procedure. Factors influencing the extraction step include fiber type, extraction time, ionic strength, sample $\mathrm{pH}$, extraction temperature and sample agitation. Variables affecting the desorption step include temperature, desorption time, focusing oven temperature, and solvent employed and its volume ${ }^{54}$. An important factor in SPME of food samples, especially in fruit and fruit juice, is matrix effect. Negative matrix effects can be reduced by diluting the samples 50- 100 fold with distilled water ${ }^{55}$.

\section{6- Matrix solid-phase dispersion (MSPD)}

MSPD is a new extraction and clean-up analysis developed for MRMs ${ }^{56}$. This technique allows the extraction of pesticides from homogeneously dispersed food samples in a solid support such as the synthetic magnesium silicate (Florisil) or silica (C8 or C18). The homomogenized matrix is placed in a column and pesticides are selectively eluted with organic solvents. Thus, sample extraction and clean-up are carried out in the same step with good recovery and reproducibility, reducing the analysis time and the amount of solvent employed. Methods based on MSPD using alumina, silica and Florisil were employed for analyses of pesticide residues in vegetables. Although recoveries using all sorbents were similar, extract from Florisil were the cleanest. Three eluting systems were used, with dichloromethane being the best ${ }^{57}$. The efficiency of different solid phases (C8, C18, cyano, amine and phenyl) for MSPD for detecting carbamate residues in fruits and vegetables using dichloromethane $(\mathrm{MeCN})$ as the solvent showed $\mathrm{C} 8$ to produce the best recovery averages of the compounds, the lowest variation in the values obtained and the cleanest extract ${ }^{58}$. MSPD has recently been used for extraction of Abamectin residues in oranges using C18-bonded silica as the sorbent and dichloromethane as the eluent; average recovery was $96 \%{ }^{59}$. Different amounts of $\mathrm{C} 18$ and silica have been used for the optimization of an MSPD method for the extraction and screening of 18 pesticides from oranges and best recoveries are obtained by using $0.5 \mathrm{~g}$ of $\mathrm{C} 18$ and $0.5 \mathrm{~g}$ of silica. The extraction efficiency of MSPD was compared with those obtained by using three classical liquid-liquid extraction methods ${ }^{60-62}$. These methods have been recommended for the analysis of organophosphorus compounds in several studies ${ }^{63-64}$. This extraction technique was also used for the quantification of organophosphorus insecticides (Malathion and Parathionmethyl) and an organochiorine pesticide ( $\beta$ - Endosulfan) in rice. Analyses of fortified rice samples were performed at different levels $(0.5-10.0 \mathrm{mg} \mathrm{kg}-1)$. Mean recoveries from three replicates ranged from $75.5 \%$ to $116.0 \%$, with coefficients of variation from $0.5 \%$ to $10.9 \%$. Kadenczki et al, ${ }^{66}$ used activated Florisil for MSPD extraction of pesticide residues from samples of plant origin. For the elution of residues (Carbamate, Organochiorine, Organophosphate, Synthetic Pyrethroid, Triazine and Phenyl Urea Pesticides) Ethyl Acetate or dichloromethane-acetone $(9: 1, \mathrm{v} / \mathrm{v})$ were used. Ling and Huang ${ }^{67}$ used Florisil-based MSPD extraction and a GC-ECD method for the determination of six synthetic pyrethroids in vegetables (West Indian gherkin, eggplant, cabbage and garden peas).

A rapid and simple method for the determination of nine organophosphorus pesticides (Diazinon, Parathion-Methyl, Fenitrothion, Malathion, Chiorpyrifos, Phenthoate, Methidathion, Profenofos and Ethion) in different fruit juices was developed on the basis of MSPD of samples on Florisil. The average recoveries obtained for all of the pesticides in the different juices and fortification levels were $>70 \%$ with relative standard deviations of $<11 \%^{68}$.

\section{7- Supercritical fluid extraction (SFE)}

Because of its high degree of selectivity and use of small amount of solvents super critical fluid extraction is being used extensively. The technique uses supercritical fluids that have similar densities to liquids, but lower viscosities and higher diffusion coefficients. This combination of properties results in a fluid that is more penetrative has a higher solvating power and extracts solutes faster than liquids ${ }^{69}$. Using automation, SFE reduces labor and laboratory space. In principle, SFE allows for a higher degree of selectivity in extraction compared with solvent-based procedures. Combined with solid sorbent traps, SFE gives a single-step extraction and clean-up ${ }^{70}$. Only moderate additional clean-up steps are required after supercritical fluid extraction. For non polar to low polar pesticides such as organochlorine and organophosphate the reported extraction efficiencies were satisfactory. The addition of polar modifiers such as methanol to $\mathrm{CO}_{2}$ increases the dissolving power of pesticides of high polarity and metabolites of pesticides. Rissato et al, ${ }^{71}$ developed the supercritical fluid extraction method for, simultaneous determination of Organophosphorus, Organohalogen, Organonitrogen and Pyrethroid pesticides in fruit and vegetables and compared it with a conventional method by GC-ECD and GCMS. Supercritical fluid extraction of pesticide residues in fortified apple matrices was done by Stefani et al, ${ }^{72}$. A collaborative study ${ }^{73}$ was conducted to determine multiple pesticide residues in apple, green bean, and carrot by using supercritical fluid extraction (SFE) and gas chromatography/mass spectrometry (GCIMS). Seventeen laboratories from 7 countries participated in the final study, and a variety of different instruments were used by collaborators. The procedure simply entailed 3 steps: (1) mixed $1.1 \mathrm{~g}$ drying agent (Hydromatrix) per $1 \mathrm{~g}$ frozen 
precomminuted sample, and loaded 4-5.5 g of this mixture into a 7-10 mm extraction vessel; (2) performed SFE for $20-30 \mathrm{~mm}$ with a $1-2 \mathrm{ml} / \mathrm{min}$ flow rate of carbon dioxide at $0.85 \mathrm{~g} / \mathrm{ml}$ density $\left(320 \mathrm{~atm}, 60^{\circ} \mathrm{C}\right)$; and (3) injected the extract, which was collected on a solid-phase or in a liquid trap, into the gas chromatograph/mass spectrometer, using either an ion-trap instrument in full-scan mode or a quadrupole-type instrument in selected-ion monitoring mode. The ability of GC/MS to simultaneously quantitate and confirm the identity of the semi volatile analytes at trace concentrations was a strong feature of the approach. The selectivity of SFE and GC/MS avoids the need for post- extraction cleanup steps, and the conversion of the $\mathrm{CO}_{2}$ solvent to a gas after SFE eliminates the solvent evaporation step common in traditional methods.

\section{8- Accelerated solvent extraction (ASE)}

This method extracts the sample under elevated temperature and pressure. It is also called as pressurized solvent extraction. It is an exceptionally effective technique with the advantage of shorter extraction times and lower consumption of solvents as compared to SOX. It allows the universal use of solvents or solvent mixtures with different polarities and individually variable pressures of 5-200 atm (0.3-20 $\mathrm{MPa})$ in order to maintain the extraction solvent in a liquid state, and temperatures ranging from room temperature up to $200^{\circ} \mathrm{C}$ in order to accelerate extraction. The solvent volume can be reduced because the solubility increases ${ }^{74}$. The use of ASE in the analysis of residual pesticides in food has been reported by a few researchers. Lehotay and Lee ${ }^{75}$ successfully used ASE to extract a broad range of pesticide residues from food matrixes for gas chromatography/ mass spectrometry (GC/MS) analysis. Obana et al, ${ }^{76}$ combined ASE, gel-permeation chromatography, and gas chromatography/flame photometric detection (GC/FPD.) to analyze residues of selected organophosphorus pesticides from food.

Adou et al, ${ }^{77}$ used ASE method and carried out single laboratory validation for 28 compounds selected from eight pesticide classes, in blank and fortified samples of fresh pear, cantaloupe, white potato, and cabbage. At each of the two fortification levels studied, 24 of the 28 pesticides gave recoveries of more than $70 \%$ with a coefficient of variation of less than $10 \%$.

\section{Conclusion}

This review includes different kind of multiresidue methods and the discussion about the use of specific chemicals which produces good recovery. For the recovery of pesticides from different matrices it is necessary to apply different types of methods which are effective, less tiring, low in cost and also it should not involve large amount of solvents and chemicals.

\section{References}

[1]. Pesticide Analytical Manual Vol. I.

M. Anastassiades, S. J. Lehotay, D. Stajnbaher and F. J. Schenck, J AOAC Int Vol. 86 No.2(2003) $412-431$.

J. Tekel and J. Shatrk, J Chromatogr. A 754 (1996) 397.

A. Ambrus and H.P. Their, Pure Appl. Chem. 58 (1986) 1036.

J.L. Tadeo, C. Sanchez-Brunete, R.A. Perez and M.D. Fernandez, J Chromatogr. A 882 (2000) 175.

L. H. Keith, and W. A. Telliard, Priority pollutants: A perspective view. Environ. Sci.Technol. 13, $416-417$ (1979).

L. H. Keith, ed., Compilation of EPA,s sampling and analysis methods, Lewis Publishers, Chelsea, MI, (1992).

W. George Fong, H. Anson Moye, James N. Seiber, John P. Toth, Pesticide Residues in Foods (Methods, Techniques, and Regulations). (1999)

[9]. R.Carabias-Martinez, E. Rodriguez-Gonzalo, P.R Paniagua- Marcos and J. Hernandez-Mendez, J Chromatogr. A 869 (2000) 427.

[10]. M. Desmarchelier, Pestic. Sci. 34(1992)215

[11]. T. Cairns, M.A. Luke, K.S. Chiu, D. Navaro and E.G. Siegmund, Rapid Commun. Mass Spectrom. 7 (1993) 1070.

[12]. A. Anderson, H. PaO lsheden, Fresenius J. Anal Chem. 339 (1991) 365.

[13]. K. Nordrneyer and Hans-Peter Their, Z Lebensm Unters Forsch A (1999) 208: 259— 263

[14]. A. de Kok and M. Hiemsfra, I AQAC mt. 75 (1992)1063.

[15]. M.J. Page and M. French, J AOAC Int.. 75 (1992) 1073.

[16]. M. Battista, A. di Corcia and M. Marchetti, Anal. Chem. 61(1989)935.

[17]. A. Lagana, A. Marino, G. Fago and B.R Martinez, Analysis 22 (1994) 63.

[18]. J. Tekel, S. Vaverkov, J. Kovaziiovfi, M. Hoilti and E. Havrinek, Pharmazie 49 (1994) 899.

[19]. F.J. Schenck and R. Wagner, Food Addit. Contain. 12 (1995) 535.

[20]. J.H. Petersen and K.G. Jensen, Z Lebensm. Unters. Forsch. 182 (1986) 489

[21]. A.J. Cessna and D.L. Benoit, Pestic: Sci. 35 (1992) 355.

[22]. A.J. Cessna, J Agric. Food Chem. 40 (1992) 1154.

[23]. A.J. Cessna, Can. J Plant Sci. 71(1991)915.

[24]. Y. Nakamura, Y. Tonogai, Y. Sekiguchi, Y. Tsumura, N. Nishida, K. Takamura, M. isechi, K. Yuasa, M. Nakamura, N. Kifune.

K. Yamamoto, S. Terasawa, T. Oshima, M. Miyata, K. Kamakura and Y. ito, J. Agric. Food Chem. 42 (1994) 2508.

H. Wan, H. Xia and Z. Chen, Food Addit. Contain. 8 (1991) 497.

H. Wan, J Chroinatogr. 516 (1990) 446.

W. Pestemer and W. Mann, 7. Lebensm. Unters. Forsch. 171 (1980) 272.

Y. Tsumura, I. Wada, Y. Fuiwara, Y. Nakamura, Y. Tonogai and Y. Ito, J Agric. Food Chem. 42 (1994) 2922.

A. Ambrus. J. Lantos, Visi, Csatios and L. Stirvflri, J.Assoc Off Anal. Chem. 64 (1981) 733.

W. Pestemer and W. Mann, 7. Lebensm. Unters. Forsch. 171 (1980) 272. 
[31]. J. Tekel, R Farkag, J. Kovaziiovfi and A. Szokolay, Nahrung 32 (1988) 357.

[32]. E Armishawand R.G. Millar, J. AOAC Int. 76 (1993) 1317

[33]. G.E. Miliadis, EA. Siskos and G.S. Vasilikiotis, .J Assoc. Off Anal Chern. 73(1990) 435.

[34]. E.M. Lores, J.C. Moore and R. Moody, Chemosphere 16 (1987) 1065.

[35]. A. Di MUccio, A. Ausili, I. Camoni, R. Dommarco, M. Rizzica and F. Vergori, J. Chromatogr. 456 (1988) 149.

[36]. M. Okihashi, Y. Kitagawa, K. Akutsu, H. Obana and Y. Tanaka, J. Pestic. Sci. 3 0(4) (2005) 368-377

[37]. J. Fillion, F. Sauvé and J. Selwyn, I. Assoc. Off Anal Chem. 83 (2000) 698.

[38]. F.J. Sehenck and V. Howard-King, Bull Environ. Contam. Toxicol 63(1999)277.

[39]. R.S. Sheridan and J.R. Meola, J Asscc Qff. Anal. Chem. 82(1999)982.

[40]. M.C. Hennion, J Chromatog A 856 (1999) 3

[41]. W.H. Newsome and P. Collins, J Chromatogr. 472 (1989) 416.

[42]. LA. Casanova, .J Assoc. Off Anal Chem. (Them. 79 (1996) 936.

[43]. C.M. Tones, Y. Picó and J. Manes, J Chromatogi A 778 (1997) 127.

[44]. K. Nordmeyer and H.-P. Thier, Z. Lebensm. Unters. Forsch. A 208 (1999) 259.

[45]. M. Battista, A. Di Corcia and M. Marchetti, Anal. Chem. 61(1989) 935.

[46]. A. Laganã, GD. Ascenzo, G. Fago and A. Marino, Chrornatographia 46 (1997) 256.

[47]. G. Niessner, W. Buchberger and O.K. Bonn, J Chromatogr. A 737 (1996) 215.

[48]. G. Niessner, W. Buchberger and R. Eckerdtorfer, . J Chromatogr r. A 846 (1999) 341.

[49]. D. Stajnbaher, L. Zupancic-Kralj, . J Chromatogr A 1015 (2003) 185-198.

[50]. M. Anastassiades, S.J. Lehotay, D. tajnbaher and FJ. Schenck, J AOAC Int. vol. 86, no.2(2003) 412-431

[51]. C. Arthur, J. Pawliszyn, Anal. Chem 62 (1990) 2145.

[52]. Y. Pico, 0. Font, J.C. Malto and J. Manes, J Chromatogr. A 885 (2000) 251.

[53]. I. Mukherjee, M. Gopal, J Chronatogr. A 754 (1996) 33.

[54]. A.L. Sirnplcio, L.V. Boas, J Chromatogr. A 833 (1999) 35

[55]. J.Sherma, J AOAC Int. 80(1997) 283.

[56]. E. Viana, J.C. Moito and J. Fouty, J. Chromatogr. A 754 (1996) 437.

[57]. M. Fernandez, Y. Pico and J. Manes, J. Chromatogr. A 871. (2000) 43.

[58]. A.I. Valenzuela,M.J. Redorido, Y. Pico and 0. Ford, J. Chromatogr. A 871 (2000) 57

[59]. P.A. Mills, J*H. Onley and R.A. Gaither, J. Assoc. Off Anal. Chem. 46 (1963)186.

[60]. A. Andersson and H. Paisheden, Fresenius J Anal.Chem. 339 (1991) 365.

[61]. A.H. Roos, A.J. Van Munsteren, F.M. Nab and L.G.M.T. Tuinstra, Anal.. Chim. Acta 196(1987)95.

[62]. V. Leoni, A.M. Caricehia and S. Chiavarini, J.Assoc. Off. Anal, Chem. Int.. 75(1992) 511

[63]. F. Hern:indez, J.M. Grases, J. Beltrin and J.V. Sancho, Chromatographia 29 (1990) 459.

[64]. W.E Cochrane, D. Chaput and J. Singh, D. Stevenson and I.D. Wilson (Editors), Analysis of pesticide and PCB residues in zater, soil and plant material, Plenum Press, New York, (1994) p. 191.

[65]. H. S. DOrea and L. Lima Sobrinho, J Braz. Chem. Soc. Vol. 15 No. 5 (2004)690-694.

[66]. L. Kadenczki, Z. Arpad, I. Girdi, Ambrus, L. Gyorfi, G Reese and W. Ebing;. J. AOAC Int.. 75 (1992) 53

[67]. Y.C. Ling and I.R. Huang.J Chromatogr. A 695 (1995) 75-82.

[68]. B. Albero, C. Saa Ncbez-Brunete and J. L. Tadeo, J Agric. Food Chern. 51(2003) 691.

[69]. K.N.T. Norman and S.H.W. Panton, J Chromatogr. A 907 (2001) 247.

[70]. J. Hajslova, C.F. Moffat and K.J. Whittle (Editors), Environmental Contaminants in Food, Sheffield Academic Press, (1999) Chapter 7, p. 215

[71]. S. R. Rissato, M. S. Gaihianea, A. G. de Souza and W M. Apon, .J Braz. Chem. Soc. Vol. 16 No. 5(2005)1038-1047.

[72]. R. Stefani, M. Buzzi ans R. Grazzi, J. Chromatogr. A 782 (1997) 123-132.

[73]. S. J. Lehotay, J AOAC Int. VoL 85, No. 5 (2002) 1148-1166.

[74]. A. Hubert, K.D. Wenzel, M. Manz, L. Weissflog, W. Engewald and G. Schuumann, Anal Chem. 72 (2000) 1294.

[75]. S. J. Lehotay and C. Lee, J. Chromatogr. A 785 (1997) 3 13-327.

[76]. H. Obana, K. Kikucbi, M. Okihashi and S. Hun, Analyst 122 (1997) 217-220.

[77]. K. Adou, W. R. Bontoyan, and P. J. Sweeney, J Agric. Food Chem. Vol. 49 No. 5 (2001) 